\title{
Pandemia do SARS-COV-2: uma revisão integrativa sobre os principais medicamentos, aprovados ou em fase de estudos, que podem ser utilizados no tratamento da COVID-19
}

\section{SARS-COV-2 PANDEMIC: an integrative review on the main drugs, approvedor understudy, that can beused in thetreatmentof COVID-19}

\author{
Hugo Mendes de Melo ${ }^{1 *}$, Maykon Jhuly Martins de Paiva $^{2} \bullet$, Ciro José Sousa de Carvalho ${ }^{3} \bullet$ \\ ${ }^{1}$ Bacharelando em Farmácia, Faculdade de Palmas, Tocantins, Brasil. ${ }^{2}$ Presidente do Conselho Regional de Farmácia do Tocantins, \\ Palmas, Tocantins, Brasil. ${ }^{3}$ Professor Adjunto, Faculdade de Palmas, Palmas, Tocantins, Brasil. *Autor para correspondência. E-mail: \\ hug.o.2@hotmail.com
}

\begin{abstract}
Resumo: Introdução: A infecção pelo SARS-CoV-2 é a maior emergência de saúde pública de importância internacional já declarada. $\mathrm{O}$ grande número de perdas de vidas se soma às dificuldades relacionadas ao manejo da doença, ainda sem tratamento eficaz disponível. Remdesivir, Azitromicina, Dexametasona, Ibuprofeno e Cloroquina são alguns dos medicamentos em fase de testes para possível utilização no tratamento contra a Covid-19. Revisão: Este estudo aborda uma revisão bibliográfica, do tipo integrativa. Para a composição deste estudo foram utilizadas publicações científicas e pronunciamentos de órgãos sanitários em português e inglês, publicados até abril de 2021. Discussão: Poucos remédios demonstraram algum efeito em combater a pandemia. Diante da ausência de medicações específicas ou vacinas em quantidade suficiente, a alternativa para evitar o colapso dos sistemas de saúde, consisti em aliar política de isolamento social a testagem da população. Considerações finais: Apesar do esforço da comunidade científica, não há um tratamento específico para bloquear a replicação viral, o que existem são medicamentos que auxiliam no tratamento dos sintomas da doença. No momento, quando há crescimento de novos casos e internações, somente a vacinação em massa terá impacto na redução de mortes e internações em intervalo curto.
\end{abstract}

Palavras-chave: covid-19, coronavírus, tratamento farmacológico.

\begin{abstract}
Introduction: SARS-CoV-2 infection is the largest public health emergency of international importance ever declared. The large number of loss of life is added to the difficulties related to the management of the disease, even with no effective treatment available. Remdesivir, Azithromycin, Dexamethasone, Ibuprofen and Chloroquine are some of the drugs under testing for possible use in the treatment against Covid-19. Review: This study addresses a bibliographic review, of the integrative type. For the composition of this study, scientific publications and pronouncements of health agencies in Portuguese and English, published until April 2021 were used. Discussion: Few drugs have shown any effect in fighting the pandemic. In the absence of specific medications or vaccines in sufficient quantity, the alternative to avoid the collapse of health systems, consisted of combining a policy of social isolation with the testing of the population. Final considerations: Despite the efforts of the scientific community, there is no specific treatment to block viral replication, there are drugs that help in the treatment of the symptoms of the disease. At the moment, when there is a growth in new cases and hospitalizations, only mass vaccination will have an impact in reducing deaths and hospitalizations in a short interval.
\end{abstract}

Keywords: covid-19, coronavirus, pharmacological treatmen.

\section{Introdução}

No fim de 2019, era noticiado por várias reportagens na imprensa mundial, o surto de um novo vírus advindo da China. A Organização Mundial de Saúde (OMS), denominou o vírus de Sars-CoV-2, apelidando-o de coronavírus por possuir uma coroa em volta de sua membrana e de COVID-19 a doença respiratória provocada pela infecção desse novo coronavírus. Em 30 de janeiro de 2020 a própria OMSA declarou que o surto dessa doença se constituiu uma emergência de saúde pública de importância mundial, caracterizandoa como uma pandemia (Garcia \& Duarte, 2020; Souza et al., 2021). A pandemia tem causado grande pressão nos sistemas de saúde mundiais, produzindo grandes impactos na saúde pública e na economia global (Melo et al., 2021). 
A infecção pelo SARS-CoV-2 tem desafiado os países e seus sistemas de saúde, sendo considerada a maior emergência de saúde pública de importância mundial já declarada. O grande número de mortes se soma às dificuldades relacionadas ao controle da doença, ainda sem tratamento eficaz disponível (Pinto et al., 2021).

No Brasil, até 08 de abril de 2021, de acordo com o Ministério da Saúde, mais de 13 milhões pessoas foram contaminadas pelo novo coronavírus. Até essa mesma data, cerca de trezentos e quarenta mil pessoas já haviam morrido em virtude das complicações da COVID-19 (BRASIL, 2021).

Os sintomas mais comuns são tosse, febre e falta de ar. O tempo entre o contágio e o início dos sintomas é, geralmente, de cinco dias, podendo variar de dois a catorze dias. Não há tratamento antiviral específico (Neto \& Pires, 2020). Nesse momento, o que mais tem preocupado a população mundial, além da rapidez de propagação da doença, é que não há nenhum medicamento específico para tratar ou prevenir o SARS-CoV-2. No entanto, governos, universidades e empresas do mundo todo estão mobilizados para combater a COVID19. As entidades estão se empenhando ao máximo para acelerar o desenvolvimento dos medicamentos experimentais e vacinas (Oliveira, 2020).

Durante epidemias, quando não há tratamentos comprovados pela ciência, geralmente usa-se medicamentos baseados em testes de laboratórios ou em estudos observacionais, no entanto, com o passar do tempo, verificou-se que fármacos que foram considerados eficazes nos estudos in vitro, nos estudos observacionais para outras patologias foram posteriormente comprovados como ineficazes em ensaios clínicos (Andrade et al., 2020).

Resultados preliminares de estudos científicos apontaram que a cloroquina e a hidroxicloroquina não são eficazes contra as formas graves da COVID-19 complicadas por pneumonia. Em um recente estudo da China, cujos resultados preliminares foram publicados, houve uma comprovação de uma elevada toxicidade dos dois medicamentos, com efeitos adversos em diversos órgãos e tecidos, e sem claro benefício (Corrêa et al., 2020). Apesar desses medicamentos serem indicados para outras patologias, o uso para COVID-19 é experimental, vindo a representar riscos à saúde devido ao risco potencial de causar reações adversas (Melo et al., 2021).

Remdesivir, Azitromicina, Dexametasona, Ibuprofeno e Cloroquina são alguns dos medicamentos em fase de testes para possível utilização no tratamento contra a Covid-19. No entanto as evidências científicas ainda são iniciais e de baixa qualidade metodológica. Não há eficácia e segurança comprovada de nenhum fármaco para infecções por coronavírus em humanos (Andrade et al., 2020).

A pandemia estabeleceu grandes desafios a todas as nações do planeta, em especial à comunidade científica internacional, que agiu de forma rápida e eficiente em várias esferas prioritárias, como por exemplo, no desenvolvimento de novos tratamentos, vacinas e diagnósticos, entre outros, apoiada em conhecimento pré-estabelecido e inovação (Ferreira \& Andricopulo, 2020).

A procura por um tratamento eficaz tornou-se o centro das ações dos pesquisadores desde o surgimento da doença. Moléculas inibidoras da replicação viral, inibidores de proteases e proteínas, inibidores da endocitose, anticorpos neutralizantes têm sido considerados e amplamente estudados nesse momento (Puga et al., 2021).

Sabe-se que o uso racional de medicamentos tem como um dos seus pilares a Medicina Baseada em Evidências e que, dessa maneira, preza pelo uso da evidência científica mais atual e completa na escolha da farmacoterapia ideal, com o melhor custo/benefício, de maneira individualizada à condição de saúde do usuário e com segurança e eficácia comprovadas em estudos adequados (Tritany \& Tritany, 2020). Desta forma, considerando que as informações de segurança dos fármacos para o tratamento da COVID-19 são escassas, esta pesquisa buscou levantar publicações científicas que evidenciam quais medicamentos podem ser sugeridos no tratamento da COVID-19.

\section{Revisão}

Este estudo foi realizado mediante uma pesquisa de revisão bibliográfica, do tipo integrativa. Para a composição deste estudo foram utilizadas publicações científicas e pronunciamentos dos órgãos sanitários Agência Nacional de Vigilância Sanitária, Ministério da Saúde e Organização Mundial de Saúde, publicados até abril de 2021. Realizou-se consultas nas bases de dados da Científic Electronic Library Online (SCIELO) e literatura Latino Americana em Ciências de Saúde (LILACS). Os critérios de inclusão das fontes bibliográficas foram trabalhos publicados no período de 2020 e 2021 e que estivessem disponíveis em texto completo, em português ou inglês. Os descritores utilizados foram COVID- 19, tratamento farmacológico e coronavírus.

O uso de medicamentos off-label, que é quando a indicação para uso diverge do que consta na bula, tem parecido uma estratégia eficiente, pois até o momento não existe intervenção farmacológica com efetividade e segurança comprovada para a COVID-19 (Braúna et al., 2021). Mesmo sem fortes evidências científicas, 
muitos medicamentos têm sido utilizados empiricamente na tentativa de diminuir a letalidade da COVID-19 (Tritany \& Tritany, 2020).

Embora não haja ainda tratamento comprovado, testes com medicamentos variados estão sendo realizados constantemente. Suas ações estão sendo monitoradas para compreensão dos mecanismos mais efetivos na luta contra a doença. Vacinas foram liberadas recentemente para uso, em busca do tratamento e cura da COVID-19 (Silva et al., 2021).

Corrêa et al. (2020) evidenciaram que um estudo sobre o uso da cloroquina e hidroxicloroquina feito em 2020 com 81 brasileiros na cidade de Manaus, teve de ser interrompido após a morte de 11 pacientes, número considerando alto para um teste clínico. Apesar das poucas evidencias científicas sobre o uso eficaz da cloroquina e hidroxicloroquina, o uso desses medicamentos ainda ocupa grande espaço no debate público, muito estimulado pelo discurso de lideranças políticas apoiados por vezes em informações de supostos estudos cuja existência é questionável.

Pacientes que utilizaram a hidroxicloroquina tiveram quase duas vezes mais chances de apresentarem reações adversas graves, quando comparados com que aqueles que não usaram este medicamento e, de uma forma mais agravada, os que fizeram o uso da cloroquina registraram quase seis vezes mais chances de apresentar reações graves, comparados aos que não usaram este medicamento (Melo et al., 2021; Braúna et al., 2021). Tritany \& Tritany (2020) evidenciaram que a hidroxicloroquina e cloroquina apresentam reações adversas e interações medicamentosas relevantes, podendo causar, por exemplo, hipoglicemia, retinopatia, arritmia e infarto.

As estatinas são medicamentos usados para controle da produção de colesterol, apoiados por comprovações cientificas, com inquestionáveis benefícios cardiovasculares. Esses medicamentos podem reduzir os mecanismos pró-inflamatórios e pró-trombóticos que caracterizam os casos mais graves de COVID19, no entanto estudos randomizados devem ser aplicados para que haja indicação desses medicamentos especificamente para prevenir complicações da infecção pelo novo coronavírus (Ferrari \& Santos, 2021).

$\mathrm{O}$ antiviral remdesivir, desde maio de 2020 recebeu a aprovação da Food and Drug Administration (FDA) para uso emergencial em pacientes graves com COVID-19, sendo expandido seu uso, no final de agosto de 2020 para o tratamento de pacientes com quadro moderado da doença (Ferreira \& Andricopulo, 2020). Estudos demonstraram que as doses intravenosas de Remdesivir foram adequadamente toleradas nos pacientes; este medicamento não foi utilizado em pacientes gravemente infectados pelo COVID-19, necessitando de ensaios clínicos de controle (Azevedo et al., 2020).

Existem várias estratégias terapêuticas para a doença COVID-19 sendo estudadas e que se centram no objetivo de reduzir significativamente o número de casos moderados ou graves. Nos casos leves, o uso do remdesivir tem se mostrado eficaz. Nos estudos, este fármaco experimental demonstrou atividade anti-vírica. Corticósteroides, uso de inibidores da interleucina-6, da interleucina-2 tem mostrado resultados em casos moderados da doença. Em casos graves têm se usado a junção destas duas modalidades terapêuticas, com o intuito de suprimir a replicação vírica e controlar a magnitude da resposta imunitária de forma a que esta seja eficaz na eliminação do vírus, causando o mínimo de danos colaterais a outros órgãos do paciente (Vilarinho \& Pereira, 2020).

Dentre as estratégias farmacológicas, a ivermectina, um medicamento anti-parasitário, tem sido amplamente recomendado por alguns profissionais de saúde para prevenção e tratamento da COVID-19. No entanto Puga et al. (2021), analisaram evidências de eficácia e segurança da ivermectina em casos de COVID19 e concluíram que não há comprovação científica, até o momento, da efetividade da ivermectina em humanos para prevenção ou tratamento da COVID-19. O aumento das prescrições de ivermectina tem sido associado aos resultados favoráveis de um estudo in vitro no impedimento da replicação do SARS-CoV-2, porém, a dose utilizada pelos pesquisadores é de aproximadamente dez vezes a aprovada por agências reguladoras como ANVISA e FDA (Tritany \& Tritany, 2020). Estudos in vivo, com aplicação de doses aceitáveis, demonstraram a ineficiência desse medicamento em relação ao COVID-19 (D’Agostini et al., 2021).

A dexametasona, um corticoide com propriedades anti-inflamatórias e imunossupressoras, usado desde os anos de 1960, nos testes clínicos evidenciou uma redução nas mortes de pacientes graves recebendo ventilação mecânica invasiva ou oxigênio, porém, não se constatou benefícios nos quadros leves de COVID19. A dexametasona não tem poder preventivo frente à infecção (Ferreira \& Andricopulo, 2020; D’Agostini et al., 2021). Medicamentos com efeitos anti-inflamatórios demonstraram eficácia no tratamento do processo de inflamação pela infecção do vírus, visto que seus mecanismos se mostraram promissores contra a COVID19. Dexametasona, anakinra, auranofina, baricitinibe e nafamostat são alguns tipos de anti-inflamatórios em fases de testes (Sales et al., 2021). 
Um medicamento em análise na China é o favipiravir, uma droga com ação comprovada contra o vírus ebola. A eficiência da droga na redução da infecção pelo SARS-CoV-2 se dá em virtude desse medicamento inibir a atividade da RNA polimerase (Sousa et al, 2020).

Apesar da OMS ter liberado seu uso, o ibuprofeno tem sido usado com cautela em pacientes com a COVID19, visto que em alguns casos, piorou a condição de saúde. Especialistas da área de saúde aconselham o uso de paracetamol, se o paciente não for alérgico, como primeira escolha em vez de ibuprofeno e outros antiinflamatórios não esteroides para tratar febre e dor de garganta, devido ao perfil de segurança já comprovado do medicamento (Oliveira \& Moraes, 2020).

Arbidol, um fármaco utilizado para profilaxia e tratamento de influenza e infecções virais respiratórias, age inibindo a fusão viral para atingir a membrana celular. Tem demonstrado atividade contra o vírus SARS, e está atualmente sendo avaliado para o tratamento de COVID-19 em vários estudos na China (Dias et al., 2020). O arbidol age por meio da inibição da ligação da proteína do envelope viral às células hospedeiras e com isso impede a entrada do vírus. Diante dessa descoberta, o medicamento foi incluído na sexta edição do plano chinês de diagnóstico e tratamento de pacientes com COVID-19 (Sousa et al., 2020).

O site ClinicalTrials é mantido pela Biblioteca Nacional de Medicina dos Estados Unidos da América, sendo esse um banco de dados de informações de registros e resultados de estudos de pesquisa clínica, é patrocinado ou financiado por várias organizações públicas e privadas em todo o mundo. Neto e Pires (2020), mapearam esse site e levantaram quais os principais medicamentos para uso na COVID-19, em fase de estudos clínicos. Os achados são detalhados a seguir.

Tabela 1. Medicamentos mais citados nos estudos clínicos.

\begin{tabular}{c}
\hline Nome da Droga \\
Hidroxicloroquina \\
Azitromicina \\
Cloroquina \\
Remdesivir \\
Dexametasona \\
Terapia padrão de COVID-19 \\
Sulfato de hidroxicloroquina \\
Lopinavir /ritonavir \\
Favipiravir \\
Nitazoxanida \\
Gás óxido nítrico \\
Colchicina \\
Colchicine Tablets \\
Fosfato de cloroquina \\
Ivermectina \\
Peginterferon Lambda-1a \\
Plasma \\
Talidomida \\
Vocilizumab (TCZ) \\
\hline Vitamina C \\
\hline
\end{tabular}

Fonte: Adaptado de Neto \& Pires (2020).

Nota-se que os medicamentos pesquisados não são apenas os antivirais, mesmo sabendo que o causador da pandemia é um vírus. A pesquisa apontou que a classe terapêutica mais investigada são os antimaláricos. Porém, nos testes clínicos descobriram que no grupo de pacientes que recebiam doses altas, havia mortes mais rápidas, precisando equilibrar a dose destes, exigindo assim um monitoramento constante, a fim de não levar o paciente acometido a uma adversidade posterior ou a óbito (Neto \& Pires, 2020). 


\section{Discussão}

Em mais de um ano de pandemia, poucos remédios demonstraram algum efeito em combatê-la. Nesse período, medicamentos falharam, outros seguem em testes, e alguns medicamentos, aparentemente, diminuem a gravidade dos casos mais graves de COVID-19. Assim, diante da ausência de medicações específicas ou vacinas em quantidade suficiente, a alternativa para evitar o colapso dos sistemas de saúde, consiste em aliar política de isolamento social a testagem da população (Souza et al., 2021).

Chefes de estado, cientistas, universidades e profissionais da saúde têm se empenhado no combate à COVID-19 em uma corrida contra o tempo. Não obstante todos os esforços, em abril de 2021, o Brasil viveu o pior momento da pandemia, com lotações em todos os hospitais e filas aguardando leitos de UTI. Alguns medicamentos têm sido apresentados como a solução no combate à doença, porém, o uso de medicamentos sem fortes evidências que comprovem seus benefícios pode gerar riscos à saúde e consequências irreversíveis para a saúde do paciente.

É de suma importância discutir o uso destes fármacos em pacientes que receberam o diagnóstico de COVID-19. Os achados da pesquisa evidenciaram que os profissionais de saúde devem analisar criteriosamente cada paciente tendo em vista as particularidades da doença e os fatores associados com o uso de medicamentos, sempre se questionando qual é a melhor opção de tratamento e se esses pacientes podem fazer o uso desta farmacoterapia.

\section{Considerações finais}

Verificou-se na bibliografia que apesar do esforço da comunidade científica, ainda não há um tratamento específico para bloquear a replicação viral, o que existem são medicamentos que auxiliam no tratamento dos sintomas da doença. Deve ser ressaltado sempre, o caráter emergencial do uso de alguns medicamentos na pandemia, pois ainda falta alternativas para o tratamento de casos mais graves. Frisa-se que pesquisas precisam continuar sendo realizadas para garantir e confirmar que os benefícios desses fármacos sejam maiores que os diversos e sérios efeitos colaterais que estão sendo evidenciados. Neste momento, quando há crescimento de novos casos e muitas internações, apenas a vacinação em massa poderá ter um impacto na redução de mortes e internações em intervalo curto. Nesse sentido, programas de vacinação são agentes poderosos e, em virtude do extraordinário progresso da ciência, já dispomos desse recurso.

\section{Referências}

Andrade, K. R. C., Carvalho, V. K. S., Farinasso, C. M., Lima, A. A., Silva, R. B, Wachira, V. K., Capucho, H. C., Souza, P. M., Vanni, T., Sachetti, C. G., \& Rêgo, D. F. 2020. Terapia medicamentosa para infecções por coronavírus em humanos: revisão sistemática rápida. Ciência \& Saúde Coletiva, 25(9), 3517-3554.

Azevedo, T. C. P., Azevedo, P. C. P., Silveira Filho, R. N., Carvalho, A. R. V. S., Cezarotti Filho, M. L., Barbosa, F. T., Sousa-Rodrigues, C. F., Matos-Rocha, T. J., \& Ramos, F. W. S. 2020. Use ofremdesivir for patientswith Covid-19: a review article. Revista da Associação Médica Brasileira, 66(6), 838-841.

Braúna, C. C., Araújo, P. M., Carvalho, R. D., Carvalho, M. G. F. M., \& Nunes, L. C. C. 2021. Farmacoeconomia aplicada ao tratamento medicamentoso para a COVID-19 em um hospital campanha. Revista Eletrônica Acervo Saúde, 13(2), 1-8.

Brasil. Ministério da Saúde. 2021. Painel Coronavírus. [Página web]. Disponível em: https://covid.saude.gov.br/.

Corrêa, M. C. D. V., Vilarinho, L., \& Barroso, W. B. G. 2020. Controvérsias em torno do uso experimental da cloroquina/hidroxicloroquina contra a Covid-19: "no magic bullet”. Physis: Revista de Saúde Coletiva, 30(2), 1-21.

D'Agostini, F. M., Favero, L., \& Silva, M. 2021. Medicamentos para o tratamento do SARS CoVI2: uma revisão bibliográfica. Revista da Semana Acadêmica do Curso de Medicina da UFFS, 4(4), 1-2.

Dias, V. M. C. H., Cunha, C. A., Vidal, C. F. L., Corradi, M. F. D. B., Michelin, L., Muglia, V., Rocha, J. L. L., Costa, S. F., Oliveira, P. R. D., Carrilho, C. M., Chebabo, A., Nunes, R. R., Diego, L. A. S., Santos, A. S., Carneiro, M., Souza Jr., A. S., Escuissato, D., Neto, C. A., Waib, L. F., Martins, R., Maurici, R., 
Chatkin, J. M., Brandão, Lobo, S. M. A., D., Oliveira, M. C., Alves, J. S., Nascimento, M. M., \& MouraNeto, J. A. 2020. Orientações sobre Diagnóstico, Tratamento e Isolamento de Pacientes com COVID 19. Journal of Infection Control, 9(2), 1-20.

Ferrari, F., \& Santos, R. D. 2021. Estatinas e COVID-19: Suspender ou não suspender? Eis a Questão! Arquivos Brasileiros de Cardiologia, 116(1), 147-152.

Ferreira, L. L. G., \& Andricopulo, A. D. 2020. Medicamentos e tratamentos para a Covid-19. Estudos Avançados, 34(100), 7-27.

Garcia, L. P., \& Duarte, E. 2020. Intervenções não farmacológicas para o enfrentamento à epidemia da COVID-19 no Brasil. Epidemiologia e Serviços de Saúde, 29(2), 1-4.

Melo, J. R. R., Duarte, E. C., Moraes, M. V., Fleck, K., Silva, A. S. N., \& Arrais, P. S. D. 2021. Reações adversas a medicamentos em pacientes com COVID-19 no Brasil: análise das notificações espontâneas do sistema de farmacovigilância brasileiro. Cadernos de Saúde Pública, 37(1), 1-17.

Neto, U. E. A., \& Pires, A. C. 2020. Drogas e medicamentos investigados para o tratamento do COVID-19. Journal of Health and Biological Sciences, 8(1), 1-7.

Oliveira, E. H. A. 2020. Coronavírus: prospecção científica e tecnológica dos fármacos em estudo para tratamento da COVID-19. Cadernos de Prospecção, 13(2), 412-423.

Oliveira, E. S., \& Morais, A. C. L. N. 2020. COVID-19: uma pandemia que alerta à população. Interamerican Journal of Medicine and Health, 3, 1-4.

Santos-Pinto, C. D. B., Miranda, E. S., \& Osorio-de-Castro, C. G. S. 2021. O “kit-covid" e o Programa Farmácia Popular do Brasil. Cadernos de Saúde Pública, 37(2), 1-5.

Person, O. C., Puga, M. E. S., Amaral, J. L. G., \& Atalla, A. N. 2021. Intervenção com ivermectina para COVID-19 (SARS-Cov 2): sinopse baseada em evidências. Disponível em: https://doi.org/10.1590/SciELOPreprints.1871.

Sales, M. V. F., Pereira, H. N. S. N., Santos, L. V. N., Lopes, M. R., \& Fonsêca, D. V. 2021. Medicamentos com propriedades anti-inflamatórias eficazes contra a covid-19: uma revisão sistemática. Journal of Biology \& Pharmacy and Agricultural Management, 17(2), 309-327.

Silva, C. C., Carvalho, C. M. O., Lima, D. C., Costa, E. S., Andrade, V. M. B., Tenorio, B. M., Britto, D. B. L. A., \& Tenorio, F. C. A. M. 2021. Covid-19: Aspectos da origem, fisiopatologia, imunologia e tratamento - uma revisão narrativa. Revista Eletrônica Acervo Saúde, 13(3), 1-8.

Sousa, M. R. N., Barros, S. S., Silva, M., Oliveira, A. P. M., Rocha, G. M., \& Oliveira, G. A. L. 2020. Patogênese e perspectivas de tratamento da Covid-19: uma revisão. Research, Society and Development, 9(7), e05973730.

Souza, A. S. R., Amorim, M. M. R., Melo, A. S. O., Delgado, A. M., Florêncio, A. C. M. C. C., Oliveira, T. V., Lira, L. C. S., Sales, L. M. S., Souza, G. A., Melo, B. C. P., Morais, I., \& Katz, L. 2021. Aspectos gerais da pandemia de COVID-19. Revista Brasileira de Saúde Materno Infantil, 21(1), 29-45.

Tritany, R. F., \&Tritany, E. F. 2020. Uso Racional de Medicamentos para COVID-19 na Atenção Primária à Saúde. Revista Saúde em Redes, 6(Supl. 2), 7-17.

Vilarinho, S., \& Pereira, J. P. 2020. COVID-19: Imunidade e Estratégicas Terapêuticas. Medicina Interna, 27(Supl. 1), 70-72.

\section{Minicurrículo}

Hugo Mendes de Melo. Bacharelando em Farmácia pela Faculdade de Palmas (FAPAL), conclusão do curso em 2021. Possui experiência na área de dispensação e distribuição de medicamentos no varejo e atacado, gestão e treinamento de equipes na área vendas de medicamentos com atuação norte e nordeste do Brasil.

Maykon Jhuly Martins de Paiva. Possui Graduação em Farmácia Generalista pelo Centro Universitário Tocantinense Presidente Antônio Carlos (UNITPAC), Aperfeiçoamento em Saúde Pública. Especialização Lato Sensu em Biotecnologia pela Universidade Católica Dom Bosco (UCDB). Especialização Lato Sensu em Farmácia Clínica e Hospitalar pela Faculdade Dom Alberto (FDA). Mestrado pelo Programa de Pós-Graduação em Ciência e Tecnologia de Alimentos da Universidade Federal do Tocantins (UFT). Atualmente é professor do curso de Farmácia e Estética e Cosmética da Faculdade de Palmas (FAPAL) e Presidente do Conselho 
Regional de Farmácia do Tocantins. Trabalha com temas relacionados a Farmácia, Farmacologia, Biotecnologia, Microbiologia, Ciência e Tecnologia de Alimentos e Saúde Pública.

Ciro José Sousa de Carvalho. Bacharel em Medicina Veterinária pela Universidade Federal do Piauí (2009), Mestre em Ciência Animal com ênfase em toxicologia pela Universidade Federal do Piauí (UFPI) com participação em Programa de Cooperação Acadêmica (PROCAD) pela Universidade Federal de Minas Gerais (UFMG) -(2012) e Doutor em Ciência Animal com ênfase em Diagnóstico precoce de metástases em câncer de mama pela Universidade Federal do Piauí (2016). Consultor "Ad hoc" de revistas nacionais indexadas. Autor e co-autor em diversas revistas indexadas nacionais e internacionais. Possui 49 citações (SCOPUS). Professor Conteudista/Tutor EAD em cursos de pós-graduação nas áreas de Saúde e Medicina Veterinária.

Como citar: Melo, H.M., Paiva, M.J.M., \& Carvalho, C.J.S. 2021. Pandemia do SARSCOV-2: uma revisão integrativa sobre os principais medicamentos, aprovados ou em fase de estudos, que podem ser utilizados no tratamento da COVID-19. Pubsaúde, 6, a151. DOI: https://dx.doi.org/10.31533/pubsaude6.a151

Recebido: 9 abr. 2021.

Revisado e aceito: 22 abr. 2021.

Conflito de interesse: os autores declaram, em relação aos produtos e companhias descritos nesse artigo, não ter interesses associativos, comerciais, de propriedade ou financeiros que representem conflito de interesse.

Licenciamento: Este artigo é publicado na modalidade Acesso Aberto sob a licença Creative Commons Atribuição 4.0 (CC-BY 4.0). 